\title{
MVN: An R Package for Assessing Multivariate Normality
}

by Selcuk Korkmaz, Dincer Goksuluk and Gokmen Zararsiz

\begin{abstract}
Assessing the assumption of multivariate normality is required by many parametric multivariate statistical methods, such as MANOVA, linear discriminant analysis, principal component analysis, canonical correlation, etc. It is important to assess multivariate normality in order to proceed with such statistical methods. There are many analytical methods proposed for checking multivariate normality. However, deciding which method to use is a challenging process, since each method may give different results under certain conditions. Hence, we may say that there is no best method, which is valid under any condition, for normality checking. In addition to numerical results, it is very useful to use graphical methods to decide on multivariate normality. Combining the numerical results from several methods with graphical approaches can be useful and provide more reliable decisions. Here, we present an R package, MVN, to assess multivariate normality. It contains the three most widely used multivariate normality tests, including Mardia's, Henze-Zirkler's and Royston's, and graphical approaches, including chi-square $\mathrm{Q}-\mathrm{Q}$, perspective and contour plots. It also includes two multivariate outlier detection methods, which are based on robust Mahalanobis distances. Moreover, this package offers functions to check the univariate normality of marginal distributions through both tests and plots. Furthermore, especially for non-R users, we provide a user-friendly web application of the package. This application is available at http://www. biosoft. hacettepe. edu. tr/MVN/.
\end{abstract}

\section{Introduction}

Many multivariate statistical analysis methods, such as MANOVA and linear discriminant analysis (MASS, Venables and Ripley (2002)), principal component analysis (FactoMineR, Husson et al. (2014), psych, Revelle (2014)), and canonical correlation (CCA, González and Déjean (2012)), require multivariate normality (MVN) assumption. If the data are multivariate normal (exactly or approximately), such multivariate methods provide more reliable results. The performances of these methods dramatically decrease if the data are not multivariate normal. Hence, researchers should check whether data are multivariate normal or not before continuing with such parametric multivariate analyses.

Many statistical tests and graphical approaches are available to check the multivariate normality assumption. Burdenski (2000) reviewed several statistical and practical approaches, including the Q-Q plot, box-plot, stem and leaf plot, Shapiro-Wilk, and Kolmogorov-Smirnov tests to evaluate univariate normality, contour and perspective plots for assessing bivariate normality, and the chi-square Q-Q plot to check multivariate normality. The author demonstrated each procedure using real data from George and Mallery (1999). Ramzan et al. (2013) reviewed numerous graphical methods for assessing both univariate and multivariate normality and showed their use in a real life problem to check the MVN assumption using chi-square and beta Q-Q plots. Holgersson (2006) stated the importance of graphical procedures and presented a simple graphical tool, which is based on the scatter plot of two correlated variables to assess whether the data belong to a multivariate normal distribution or not. Svantesson and Wallace (2003) applied Royston's and Henze-Zirkler's tests to multiple-input multiple-output data to test MVN. According to the review by Mecklin and Mundfrom (2005), more than fifty statistical methods are available for testing MVN. They conducted a comprehensive simulation study based on type I and type II error and concluded that no single test excelled in all situations. The authors suggested using Henze-Zirkler's and Royston's tests among others for assessing MVN because of their good type I error control and power. Moreover, to diagnose the reason for deviation from multivariate normality, the authors suggested the use of Mardia's multivariate skewness and kurtosis statistics test as well as graphical approaches such as the chi-square Q-Q plot. Deciding which test to use can be a daunting task for researchers (mostly for non-statisticians) and it is very useful to perform several tests and examine the graphical methods simultaneously. Although there are a number of studies describing multifarious approaches, there is no single easy-to-use, up-to-date and comprehensive tool to apply various statistical tests and graphical methods together at present.

In this paper, we introduce an R package, MVN (Korkmaz et al., 2014), which implements the three most widely used MVN tests, including Mardia's, Henze-Zirkler's, and Royston's. In addition to statistical tests, the MVN also provides some graphical approaches such as chi-square Q-Q, perspective, and contour plots. Moreover, this package includes two multivariate outlier detection methods, which are based on Mahalanobis distance. In addition to multivariate normality, users can also check univariate normality tests and plots to diagnose deviation from normality via package version 3.7 and later. First, we discuss the theoretical background on the corresponding MVN tests. Second, two illustrative examples are presented in order to demonstrate the applicability of the package. Finally, 
we present a newly developed web interface of the MVN package, which can be especially handy for non-R users. The R version of MVN is publicly available from the Comprehensive R Archive Network (CRAN, http: //CRAN. R-project.org/package=MVN).

\section{Multivariate normality tests}

\section{Mardia's MVN test}

Mardia (1970) proposed a multivariate normality test which is based on multivariate extensions of skewness $\left(\hat{\gamma}_{1, p}\right)$ and kurtosis $\left(\hat{\gamma}_{2, p}\right)$ measures as follows:

$$
\hat{\gamma}_{1, p}=\frac{1}{n^{2}} \sum_{i=1}^{n} \sum_{j=1}^{n} m_{i j}^{3} \quad \text { and } \quad \hat{\gamma}_{2, p}=\frac{1}{n} \sum_{i=1}^{n} m_{i i}^{2}
$$

where $m_{i j}=\left(x_{i}-\bar{x}\right)^{\prime} S^{-1}\left(x_{j}-\bar{x}\right)$, the squared Mahalanobis distance, and $p$ is the number of variables. The test statistic for skewness, $(n / 6) \hat{\gamma}_{1, p}$, is approximately $\chi^{2}$ distributed with $p(p+1)(p+2) / 6$ degrees of freedom. Similarly, the test statistic for kurtosis, $\hat{\gamma}_{2, p}$, is approximately normally distributed with mean $p(p+2)$ and variance $8 p(p+2) / n$.

For small samples, the power and the type I error could be violated. Therefore, Mardia (1974) introduced a correction term into the skewness test statistic, usually when $n<20$, in order to control type I error. The corrected skewness statistic for small samples is $(n k / 6) \hat{\gamma}_{1, p}$, where $k=$ $(p+1)(n+1)(n+3) /(n(n+1)(p+1)-6)$. This statistic is also distributed as $\chi^{2}$ with degrees of freedom $p(p+1)(p+2) / 6$.

\section{Henze-Zirkler's MVN test}

The Henze-Zirkler's test is based on a non-negative functional distance that measures the distance between two distribution functions. If data are distributed as multivariate normal, the test statistic is approximately log-normally distributed. First, the mean, variance, and smoothness parameter are calculated. Then, the mean and the variance are log-normalized and the p-value is estimated (Henze and Zirkler, 1990; Johnson and Wichern, 1992; Henze and Wagner, 1997; Mecklin and Mundfrom, 2003; Alpar, 2013). The test statistic of Henze-Zirkler's multivariate normality test is given in equation 2.

$$
H Z=\frac{1}{n} \sum_{i=1}^{n} \sum_{j=1}^{n} e^{-\frac{\beta^{2}}{2} D_{i j}}-2\left(1+\beta^{2}\right)^{-\frac{p}{2}} \sum_{i=1}^{n} e^{-\frac{\beta^{2}}{2\left(1+\beta^{2}\right)} D_{i}}+n\left(1+2 \beta^{2}\right)^{-\frac{p}{2}}
$$

where

$$
\begin{aligned}
p & : \text { number of variables } \\
\beta & =\frac{1}{\sqrt{2}}\left(\frac{n(2 p+1)}{4}\right)^{\frac{1}{p+4}} \\
D_{i j} & =\left(x_{i}-x_{j}\right)^{\prime} S^{-1}\left(x_{i}-x_{j}\right) \\
D_{i} & =\left(x_{i}-\bar{x}\right)^{\prime} S^{-1}\left(x_{i}-\bar{x}\right)=m_{i i}
\end{aligned}
$$

From equation 2, $D_{i}$ gives the squared Mahalanobis distance of $i^{\text {th }}$ observation to the centroid and $D_{i j}$ gives the Mahalanobis distance between $i^{\text {th }}$ and $j^{\text {th }}$ observations. If data are multivariate normal, the test statistic (HZ) is approximately log-normally distributed with mean $\mu$ and variance $\sigma^{2}$ as given below:

$$
\begin{aligned}
\mu= & -\frac{a^{-\frac{p}{2}}\left(1+p \beta^{\frac{2}{a}}+\left(p(p+2) \beta^{4}\right)\right)}{2 a^{2}} \\
\sigma^{2}= & 2\left(1+4 \beta^{2}\right)^{-\frac{p}{2}}+\frac{2 a^{-p}\left(1+2 p \beta^{4}\right)}{a^{2}}+\frac{3 p(p+2) \beta^{8}}{4 a^{4}} \\
& -4 w_{\beta}{ }^{-\frac{p}{2}}\left(1+\frac{3 p \beta^{4}}{2 w_{\beta}}+\frac{p(p+2) \beta^{8}}{2 w_{\beta^{2}}^{2}}\right)
\end{aligned}
$$

where $a=1+2 \beta^{2}$ and $w_{\beta}=\left(1+\beta^{2}\right)\left(1+3 \beta^{2}\right)$. Hence, the log-normalized mean and variance of the 
$H Z$ statistic can be defined as follows:

$$
\log (\mu)=\log \left(\sqrt{\frac{\mu^{4}}{\sigma^{2}+\mu^{2}}}\right) \quad \text { and } \quad \log \left(\sigma^{2}\right)=\log \left(\frac{\sigma^{2}+\mu^{2}}{\sigma^{2}}\right)
$$

By using the log-normal distribution parameters, $\mu$ and $\sigma$, we can test the significance of multivariate normality. The Wald test statistic for multivariate normality is given in equation 4 .

$$
z=\frac{\log (H Z)-\log (\mu)}{\log (\sigma)}
$$

\section{Royston's MVN test}

Royston's test uses the Shapiro-Wilk/Shapiro-Francia statistic to test multivariate normality. If kurtosis of the data is greater than 3, then it uses the Shapiro-Francia test for leptokurtic distributions, otherwise it uses the Shapiro-Wilk test for platykurtic distributions (Shapiro and Wilk, 1964; Royston, 1982, 1983, 1992; Johnson and Wichern, 1992; Royston, 1995; Mecklin and Mundfrom, 2005).

Let $W_{j}$ be the Shapiro-Wilk/Shapiro-Francia test statistic for the $j^{\text {th }}$ variable $(j=1,2, \ldots, p)$ and $Z_{j}$ be the values obtained from the normality transformation proposed by Royston (1992).

$$
\begin{array}{llll}
\text { if } 4 \leq n \leq 11 ; & x=n & \text { and } & w_{j}=-\log \left[\gamma-\log \left(1-W_{j}\right)\right] \\
\text { if } 12 \leq n \leq 2000 ; & x=\log (n) & \text { and } & w_{j}=\log \left(1-W_{j}\right)
\end{array}
$$

As seen from equation $5, x$ and $w_{j}$-s change with the sample size $(n)$. By using equation 5 , transformed values of each random variable can be obtained from equation 6 .

$$
Z_{j}=\frac{w_{j}-\mu}{\sigma}
$$

where $\gamma, \mu$ and $\sigma$ are derived from the polynomial approximations given in equation 7 . The polynomial coefficients are provided by Royston (1992) for different sample sizes.

$$
\begin{aligned}
\gamma & =a_{0 \gamma}+a_{1 \gamma} x+a_{2 \gamma} x^{2}+\cdots+a_{d \gamma} x^{d} \\
\mu & =a_{0 \mu}+a_{1 \mu} x+a_{2 \mu} x^{2}+\cdots+a_{d \mu} x^{d} \\
\log (\sigma) & =a_{0 \sigma}+a_{1 \sigma} x+a_{2 \sigma} x^{2}+\cdots+a_{d \sigma} x^{d}
\end{aligned}
$$

The Royston's test statistic for multivariate normality is as follows:

$$
H=\frac{e \sum_{j=1}^{p} \psi_{j}}{p} \sim \chi_{e}^{2}
$$

where $e$ is the equivalent degrees of freedom (edf) and $\Phi(\cdot)$ is the cumulative distribution function for the standard normal distribution such that,

$$
\begin{aligned}
e & =p /[1+(p-1) \bar{c}] \\
\psi_{j} & =\left\{\Phi^{-1}\left[\Phi\left(-Z_{j}\right) / 2\right]\right\}^{2}, \quad j=1,2, \ldots, p .
\end{aligned}
$$

As seen from equation 9, another extra term $\bar{c}$ has to be calculated in order to continue with the statistical significance of Royston's test statistic given in equation 8 . Let $R$ be the correlation matrix and $r_{i j}$ be the correlation between $i^{\text {th }}$ and $j^{\text {th }}$ variables. Then, the extra term $\bar{c}$ can be found by using equation 10.

$$
\bar{c}=\sum_{i} \sum_{j} \frac{c_{i j}}{p(p-1)}, \quad\left\{c_{i j}\right\}_{i \neq j}
$$

where

$$
c_{i j}= \begin{cases}g\left(r_{i j}, n\right) & \text { if } i \neq j \\ 1 & \text { if } i=j\end{cases}
$$

with the boundaries of $g(\cdot)$ as $g(0, n)=0$ and $g(1, n)=1$. The function $g(\cdot)$ is defined as follows:

$$
g(r, n)=r^{\lambda}\left[1-\frac{\mu}{v}(1-r)^{\mu}\right]
$$

The unknown parameters $\mu, \lambda$, and $v$ were estimated from a simulation study conducted by Ross (1980). He found $\mu=0.715$ and $\lambda=5$ for sample size $10 \leq n \leq 2000$ and $v$ is a cubic function which 
can be obtained as follows:

$$
v(n)=0.21364+0.015124 x^{2}-0.0018034 x^{3}
$$

where $x=\log (n)$.

\section{Implementation of MVN package}

The MVN package contains several functions in the S4 class system. The data to be analyzed should be given as a "data.frame" or "matrix" object. In this example, we will work with the famous Iris data set. These data are from a multivariate data set introduced by Fisher (1936) as an application of linear discriminant analysis. It is also called Anderson's Iris data set because Edgar Anderson collected the data to measure the morphologic variation of Iris flowers of three related species (Anderson, 1936). First of all, the MVN package should be loaded in order to use related functions.

\# load MVN package

library(MVN)

Similarly, the Iris data can be loaded from the R database by using the following R code:

\# load Iris data

data(iris)

The Iris data set consists of 150 samples from each of the three species of Iris including setosa, virginica, and versicolor. For each sample, four variables were measured including the length and width of the sepals and petals, in centimeters.

Example I: For simplicity, we will work with a subset of these data which contain only 50 samples of setosa flowers, and check MVN assumption using Mardia's, Royston's and Henze-Zirkler's tests.

\# setosa subset of the Iris data

setosa <- iris $[1: 50,1: 4]$

\section{Mardia's MVN test: mardiaTest(...)}

The mardiaTest function is used to calculate the Mardia's test multivariate skewness and kurtosis coefficients as well as their corresponding statistical significance. This function can also calculate the corrected version of the skewness coefficient for small sample size $(n<20)$.

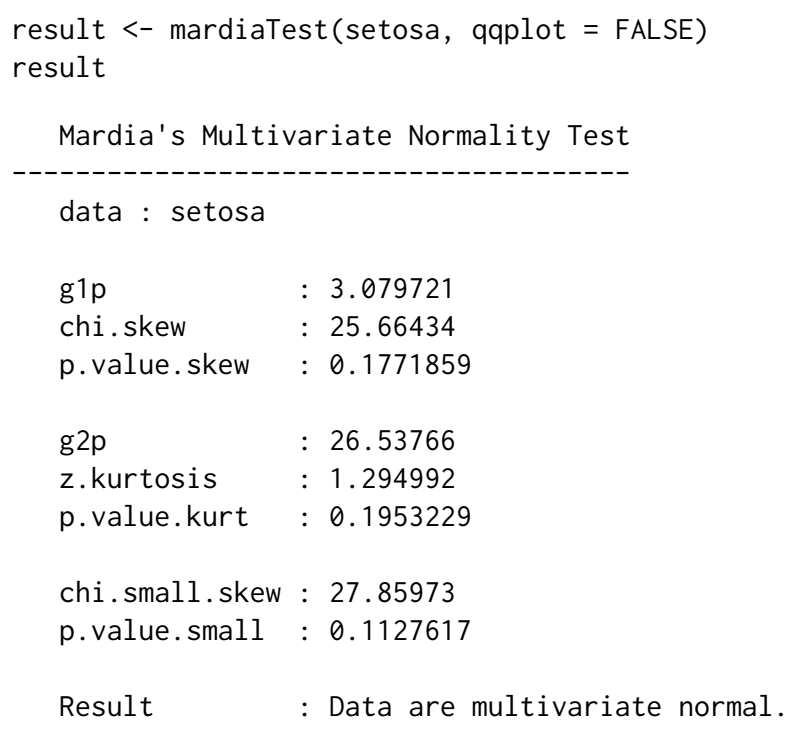

Here:

g1p: Mardia's estimate of multivariate skewness, i.e $\hat{\gamma}_{1, p}$ given in equation 1, chi.skew: test statistic for multivariate skewness,

p. value. skew: significance value of skewness statistic, 
g2p: Mardia's estimate of multivariate kurtosis, i.e $\hat{\gamma}_{2, p}$ given in equation 1 , z. kurtosis: test statistic for multivariate kurtosis, p. value. kurt: significance value of kurtosis statistic, chi.small. skew: test statistic for multivariate skewness with small sample correction, p.value.small: significance value of small sample skewness statistic.

As seen from the results given above, both the skewness $\left(\hat{\gamma}_{1, p}=3.0797, p=0.1772\right)$ and kurtosis $\left(\hat{\gamma}_{2, p}=26.5377, p=0.1953\right)$ estimates indicate multivariate normality. Therefore, according to Mardia's MVN test, this data set follows a multivariate normal distribution.

\section{Henze-Zirkler's MVN test: hzTest(...)}

One may use the hzTest function in the MVN package to perform the Henze-Zirkler's test.

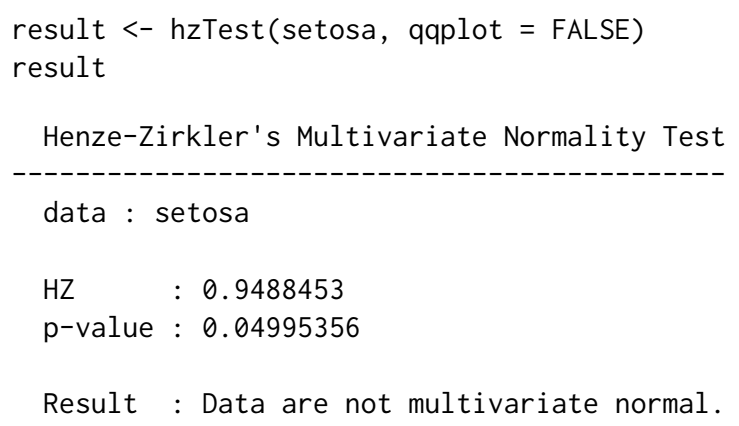

Here, $\mathrm{HZ}$ is the value of the Henze-Zirkler's test statistic at significance level 0.05 and p-value is the significance value of this test statistic, i.e., the significance of multivariate normality. As the p-value, which is derived from hzTest, is mathematically lower than 0.05 , one can conclude that this multivariate data set deviates slightly from multivariate normality $(H Z=0.9488, p=0.04995)$. As the p-value is very close to 0.05 , researchers should also check the multivariate graphical approaches as well as univariate tests and plots to make a more reliable decision on multivariate normality.

\section{Royston's MVN test: roystonTest(...)}

In order to carry out the Royston's test, roystonTest function in the MVN package can be used as follows:

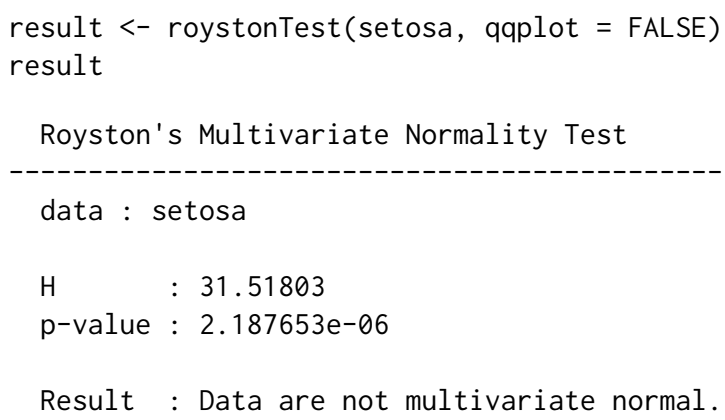

Here, $\mathrm{H}$ is the value of the Royston's test statistic at significance level 0.05 and $\mathrm{p}$-value is an approximate significance value for the test with respect to edf. According to Royston's test, the setosa data set does not appear to follow a multivariate normal distribution $(H=31.518, p<0.001)$.

\section{Chi-square Q-Q plot}

One can clearly see that different MVN tests may come up with different results. MVN assumption was rejected by Henze-Zirkler's and Royston's tests; however, it was not rejected by Mardia's test at a significance level of 0.05 . In such cases, examining MVN plots along with hypothesis tests can be quite useful in order to reach a more reliable decision. 
The Q-Q plot, where “Q" stands for quantile, is a widely used graphical approach to evaluate the agreement between two probability distributions. Each axis refers to the quantiles of probability distributions to be compared, where one of the axes indicates theoretical quantiles (hypothesized quantiles) and the other indicates the observed quantiles. If the observed data fit hypothesized distribution, the points in the Q-Q plot will approximately lie on the line $y=x$.

MVN has the ability to create three multivariate plots. One may use the qqplot = TRUE option in the mardiaTest, hzTest, and roystonTest functions to create a chi-square Q-Q plot. We can create this plot for the setosa data set to see whether there are any deviations from multivariate normality. Figure 1 shows the chi-square Q-Q plot of the first 50 rows of the Iris data, which are setosa flowers. It can be seen from Figure 1 that there are some deviations from the straight line and this indicates possible departures from a multivariate normal distribution.

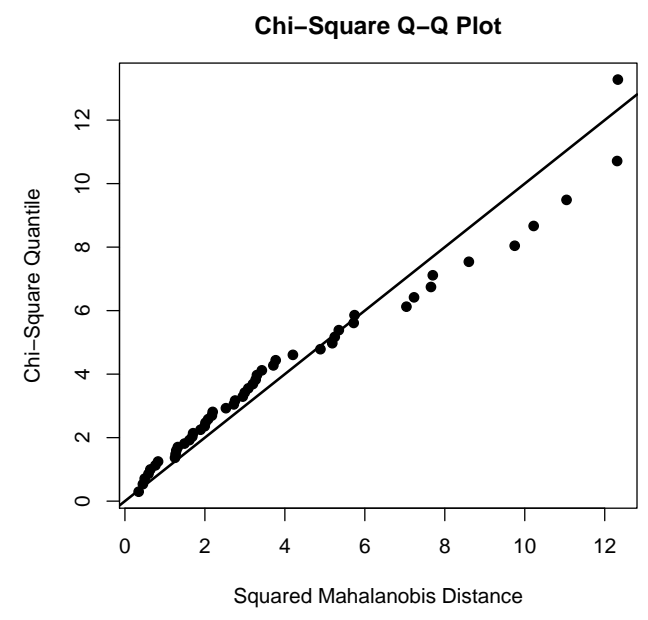

Figure 1: Chi-Square $\mathrm{Q}-\mathrm{Q}$ plot for setosa data set.

As a result, we can conclude that this data set does not satisfy MVN assumption based on the fact that the two test results are against it and the chi-square Q-Q plot indicates departures from multivariate normal distribution.

\section{Univariate plots and tests}

As noted by several authors (Burdenski, 2000; Stevens, 2012; Kass et al., 2014), if data have a multivariate normal distribution, then, each of the variables has a univariate normal distribution; but the opposite does not have to be true. Hence, checking univariate plots and tests could be very useful to diagnose the reason for deviation from MVN. We can check this assumption through uniPlot and uniNorm functions from the package. The uniPlot function is used to create univariate plots, such as Q-Q plots (Figure 2a), histograms with normal curves (Figure 2b), box-plots and scatterplot matrices.

uniPlot (setosa, type = "qqplot") \# draw univariate Q-Q plots

uniPlot(setosa, type = "histogram") \# draw univariate histograms

As seen from Figure 2, Petal. Width has a right-skewed distribution whereas other variables have approximately normal distributions. Thus, we can conclude that problems with multivariate normality arise from the skewed distribution of Petal.Width. In addition to the univariate plots, one can also perform univariate normality tests using the uniNorm function. It provides several widely used univariate normality tests, including Shapiro-Wilk, Cramer-von Mises, Lilliefors, and AndersonDarling. For example, the following code chunk is used to perform the Shapiro-Wilk's normality test on each variable:

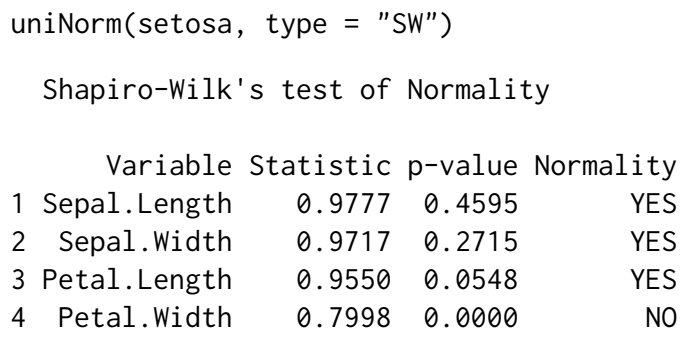



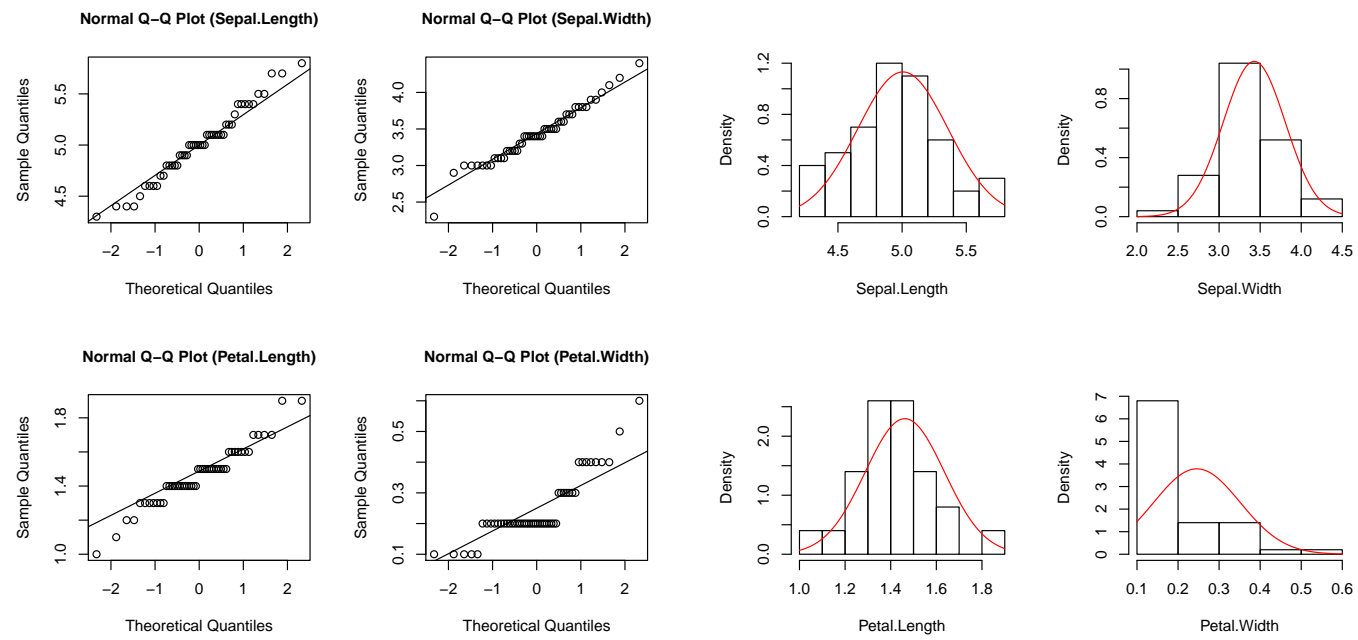

(a) Q-Q plots.

(b) Histograms with normal curves.

Figure 2: Univariate plots of setosa.

From the above results, we can see that all variables, except Petal.Width in the setosa data set, have univariate normal distributions at significance level 0.05. We can now drop Petal.With from setosa data and recheck the multivariate normality. MVN results are given in Table 1.

\begin{tabular}{lrr}
\hline Test & Test Statistic & p-value \\
\hline Mardia & & \\
Skewness & 11.249 & 0.338 \\
Kurtosis & 1.287 & 0.198 \\
Henze-Zirkler & 0.524 & 0.831 \\
Royston & 7.255 & 0.060 \\
\hline
\end{tabular}

Table 1: MVN test results (setosa without Petal.Width).

According to the three MVN test results in Table 1, setosa without Petal.Width has a multivariate normal distribution at significance level 0.05 .

Example II: Whilst the Q-Q plot is a general approach for assessing MVN in all types of numerical multivariate datasets, perspective and contour plots can only be used for bivariate data. To demonstrate the applicability of these two approaches, we will use a subset of Iris data, named setosa2, including the sepal length and sepal width variables of the setosa species.

\section{Perspective and contour plots}

Univariate normal marginal densities are a necessary but not a sufficient condition for MVN. Hence, in addition to univariate plots, creating perspective and contour plots will be useful. The perspective plot is an extension of the univariate probability distribution curve into a 3-dimensional probability distribution surface related with bivariate distributions. It also gives information about where data are gathered and how two variables are correlated with each other. It consists of three dimensions where two dimensions refer to the values of the two variables and the third dimension, which like in univariate cases, is the value of the multivariate normal probability density function. Another alternative graph, which is called the "contour plot", involves the projection of the perspective plot into a 2-dimensional space and this can be used for checking multivariate normality assumption. For bivariate normally distributed data, we expect to obtain a three-dimensional bell-shaped graph from the perspective plot. Similarly, in the contour plot, we can observe a similar pattern.

To construct a perspective and contour plot for Example 2, we can use the mvnPlot function in MVN. This function requires an object of the "MVN" class that is the result from one of the MVN functions. In the following codes, the object from hzTest is used for the perspective plot given in Figure 3a. It is also possible to create a contour plot of the data. Contour graphs are very useful as they give information about normality and correlation at the same time. Figure $3 b$ shows the contour plot of setosa flowers. As can be seen from the graph, this is simply a top view of the perspective plot where 
the third dimension is represented with ellipsoid contour lines. From this graph, we can say that there is a positive correlation among the sepal measures of flowers since the contour lines lie around the main diagonal. If the correlation were zero, the contour lines would be circular rather than ellipsoid.

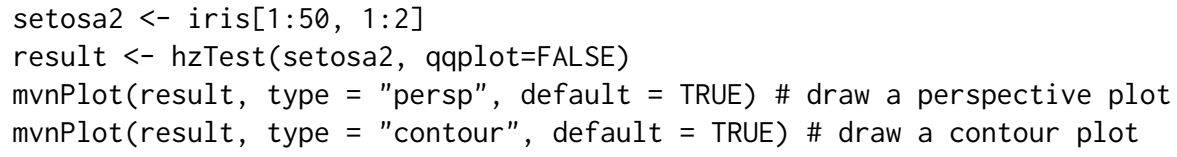

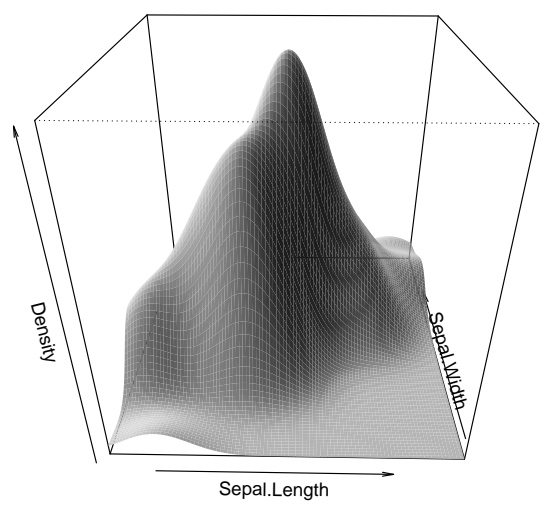

(a) Perspective plot

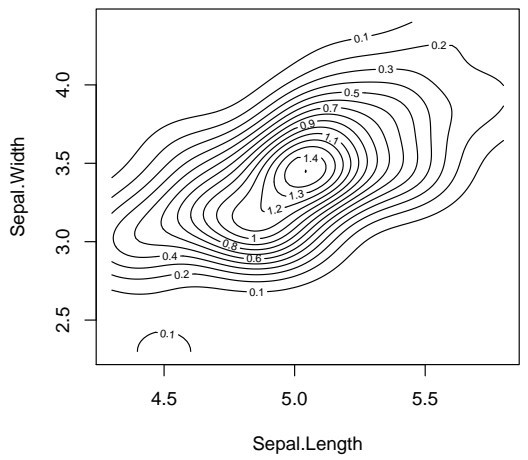

(b) Contour plot

Figure 3: Perspective and contour plot for bivariate setosa2 data set.

As neither the univariate plots in Figure 2 nor the multivariate plots in Figure 3 show any significant deviation from MVN, we can now perform the MVN tests to evaluate the statistical significance of bivariate normal distribution of the setosa2 data set.

\begin{tabular}{lrr}
\hline Test & Test Statistic & p-value \\
\hline Mardia & & \\
Skewness & 0.760 & 0.944 \\
Kurtosis & 0.093 & 0.926 \\
Henze-Zirkler & 0.286 & 0.915 \\
Royston & 2.698 & 0.245 \\
\hline
\end{tabular}

Table 2: MVN test results (setosa with sepal measures).

All three tests in Table 2 indicate that the data set satisfies bivariate normality assumption at the significance level 0.05. Moreover, the perspective and contour plots are in agreement with the test results and indicate approximate bivariate normality.

Figures $3 \mathrm{a}$ and $3 \mathrm{~b}$ were drawn using a pre-defined graphical option by the authors. However, users may change these options by setting function entry to default = FALSE. If the default is FALSE, optional arguments from the plot, persp and contour functions may be introduced to the corresponding graphs.

\section{Multivariate outliers}

Multivariate outliers are the common reason for violating MVN assumption. In other words, MVN assumption requires the absence of multivariate outliers. Thus, it is crucial to check whether the data have multivariate outliers, before starting multivariate analysis. The MVN includes two multivariate outlier detection methods which are based on robust Mahalanobis distances $(\operatorname{rMD}(x))$. Mahalanobis distance is a metric which calculates how far each observation is to the center of joint distribution, which can be thought of as the centroid in multivariate space. Robust distances are estimated from minimum covariance determinant estimators rather than the sample covariance (Rousseeuw and Leroy, 1987). These two approaches, defined as Mahalanobis distance and adjusted Mahalanobis distance in the package, detect multivariate outliers as given below, 
Mahalanobis Distance:

1. Compute robust Mahalanobis distances $\left(\operatorname{rMD}\left(x_{i}\right)\right)$,

2. Compute the 97.5 percent quantile $(Q)$ of the chi-square distribution,

3. Declare $\operatorname{rMD}\left(x_{i}\right)>Q$ as possible outlier.

Adjusted Mahalanobis Distance:

1. Compute robust Mahalanobis distances $\left(\operatorname{rMD}\left(x_{i}\right)\right)$,

2. Compute the 97.5 percent adjusted quantile $(A Q)$ of the chi-Square distribution,

3. Declare $\operatorname{rMD}\left(x_{i}\right)>A Q$ as possible outlier.

The mvOutlier function is used to detect multivariate outliers as given below. It also returns a new data set in which declared outliers are removed. Moreover, Q-Q plots can be created by setting qqplot $=$ TRUE within mvOutlier for visual inspection of the possible outliers. For this example, we will use another subset of the Iris data, which is versicolor flowers, with the first three variables.

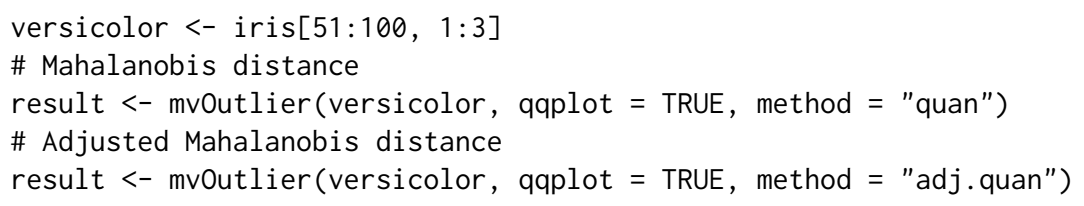

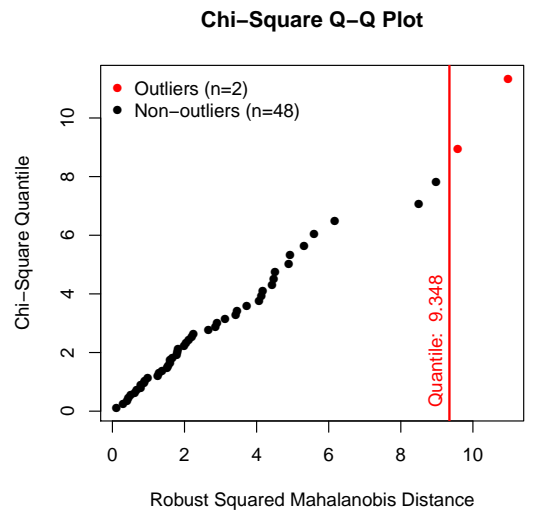

(a) Mahalanobis Distance

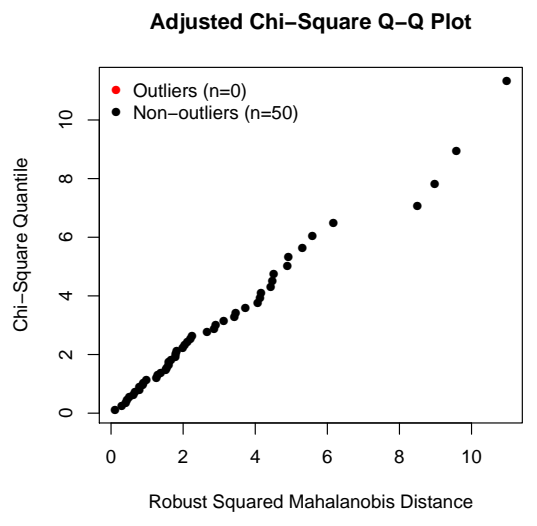

(b) Adjusted-Mahalanobis Distance

Figure 4: Multivariate outlier detection.

From Figure 4, Mahalanobis distance declares 2 observations as multivariate outlier whereas adjusted Mahalanobis distance declares none. See Filzmoser et al. (2005) for further information on multivariate outliers.

\section{Web interface for the MVN package}

The purpose of the package is to provide MVN tests along with graphical approaches for assessing MVN. Moreover, this package offers univariate tests and plots, and multivariate outlier detection for checking MVN assumptions through R. However, using R codes might be challenging for new R users. Therefore, we also developed a user-friendly web application of MVN by using shiny ${ }^{1}$ (RStudio, Inc., 2014), and this is publicly available. ${ }^{2}$ This web-tool, which is an interactive application, has all the features that the MVN package has.

To start analysis, users need to upload or paste their data to the web-tool as described in the Data upload tab. Three example data sets are available on this page for researchers to test the tool and prepare their data in an appropriate form (Figure 5a). Before performing MVN tests, the univariate normality assumption can be checked through univariate plots (Q-Q plot, histograms with normal curve, box-plot, and scatterplot matrix) and tests (Shapiro-Wilk, Cramer-von Mises, Lilliefors, and

\footnotetext{
$1_{\text {http: //www.rstudio.com/shiny/ }}$

${ }^{2}$ http: //www. biosoft. hacettepe.edu. tr/MVN
} 


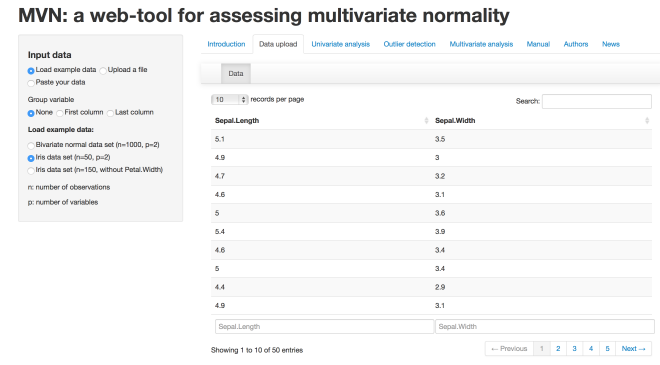

(a) Data upload

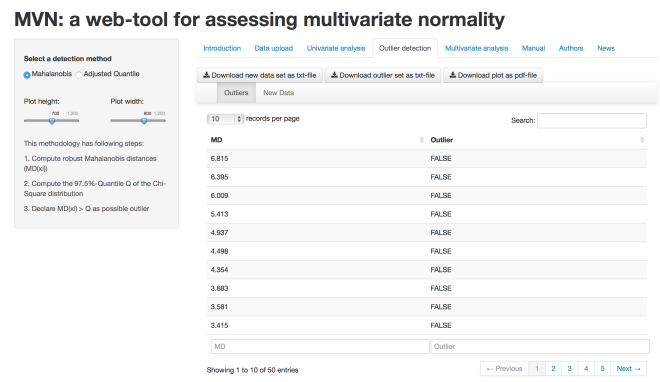

(c) Outlier detection

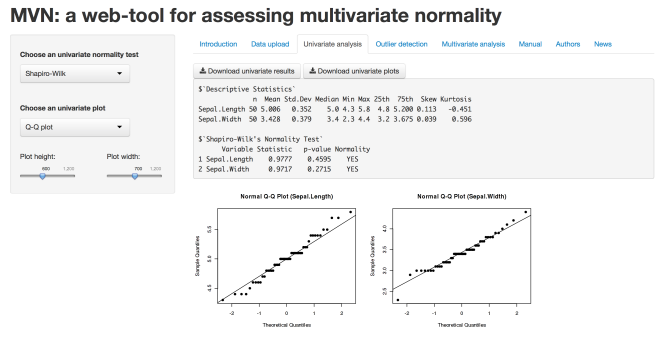

(b) Univariate analysis

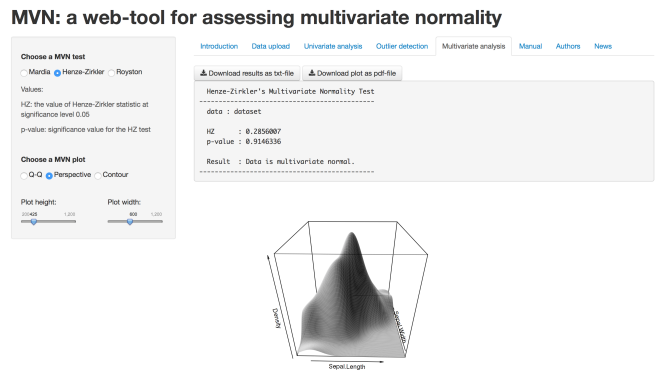

(d) Multivariate analysis

Figure 5: Web interface of the MVN package.

Anderson-Darling) in the Univariate analysis tab (Figure 5b) and possible multivariate outliers can be detected by using the Mahalanobis distances via the Outlier detection tab as seen in Figure $5 c$. Finally, users can assess MVN in the Multivariate analysis tab by choosing one of the MVN tests including Mardia's, Henze-Zirkler's, and Royston's and graphical approaches, including chi-square Q-Q, perspective, and contour plots (Figure $5 \mathrm{~d}$ ). All the results can be downloaded by using download buttons in the respective tabs.

\section{Summary and further research}

As stated earlier, MVN is among the most crucial assumptions for most parametric multivariate statistical procedures. The power of these procedures is negatively affected if this assumption is not satisfied. Thus, before using any of the parametric multivariate statistical methods, MVN assumption should be tested first. Although there are many MVN tests, there is not a standard test for assessing this assumption. In our experience, researchers may choose Royston's test for data with a small sample size $(n<50)$ and Henze-Zirkler's test for a large sample size $(n>100)$. However, a more comprehensive simulation study is needed to provide more reliable inference. Instead of using just one test, it is suggested that using several tests simultaneously and examining some graphical representation of the data may be more appropriate. Currently, as far as we know, there is no such extensive tool to apply different statistical tests and graphical methods together.

In this paper, we present the MVN package for multivariate normality checking. This package offers comprehensive flexibility for assessing MVN assumption. It contains the three most widely used MVN tests, including Mardia's, Henze-Zirkler's and Royston's. Moreover, researchers can create three MVN plots using this package, including the chi-square Q-Q plot for any data set and perspective and contour plots for bivariate data sets. Furthermore, since MVN requires univariate normality of each variable, users can check univariate normality assumption by using both univariate normality tests and plots with proper functions in the package. In the first example, different results on multivariate normality were achieved from the same data. When sepal and petal measures, i.e., four variables, were considered, Mardia's test resulted in multivariate normality as well as Henze-Zirkler's test at the edge of type I error. However, Royston's test strongly rejected the null hypothesis in favor of non-normality. At this point, the only possible graphical approach is to use the chi-square Q-Q plot since there are more than two variables. The next step was to identify the cause of deviation from MVN by using univariate normality tests and plots. In the second example, all tests suggested bivariate normality, as did the graphical approaches. Although some tests may not reject the null hypothesis, other tests may reject it. Hence, as stated earlier, selecting the appropriate MVN test dramatically changes the results and the final decision is ultimately the researcher's. 
Currently, MVN works with several statistical tests and graphical approaches. It will continue to add new statistical approaches as they are developed. The package and the web-tool will be regularly updated based on these changes.

\section{Acknowledgments}

We would like to thank Prof. Dr. C. Reha Alpar and the anonymous reviewer for their very useful comments and suggestions which helped us to improve the quality of our paper. We would also like to thank Izzet Parug Duru from Marmara University Department of Physics and Vahap Eldem from Istanbul University Department of Biology for making the web-tool version of the package possible. This study was supported by the Research Fund of Marmara University [FEN-C-DRP-120613-0273].

\section{Bibliography}

R. Alpar. Uygulamalı Çok Değişkenli İstatistiksel Yöntemler. Detay Yayıncılık, Ankara, Turkey, fourth edition, 2013. ISBN 978-605-5437-42-8. [p152]

E. Anderson. The species problem in Iris. Missouri Botanical Garden Press, 23(3):457-509, 1936. [p154]

T. Burdenski. Evaluating univariate, bivariate, and multivariate normality using graphical and statistical procedures. Multiple Linear Regression Viewpoints, 26(2):15-28, 2000. [p151, 156]

P. Filzmoser, R. G. Garrett, and C. Reimann. Multivariate outlier detection in exploration geochemistry. Computers $\mathcal{E}$ Geosciences, 31(5):579-587, 2005. [p159]

R. A. Fisher. The use of multiple measurements in taxonomic problems. Annals of Eugenics, 7(2): 179-188, 1936. [p154]

D. George and P. Mallery. SPSS for Windows step by step. Allyn \& Bacon, Boston, 1999. [p151]

I. González and S. Déjean. CCA: Canonical correlation analysis, 2012. URL http://CRAN . R-project. org/package=CCA. R package version 1.2. [p151]

N. Henze and T. Wagner. A new approach to the BHEP tests for multivariate normality. Journal of Multivariate Analysis, 62(1):1-23, 1997. [p152]

N. Henze and B. Zirkler. A class of invariant consistent tests for multivariate normality. Communications in Statistics - Theory and Methods, 19(10):3595-3617, 1990. [p152]

H. Holgersson. A graphical method for assessing multivariate normality. Computational Statistics, 21 (1):141-149, 2006. [p151]

F. Husson, J. Josse, S. Le, and J. Mazet. FactoMineR: Multivariate Exploratory Data Analysis and Data Mining with $R$, 2014. URL http: //CRAN. R-project. org/package=FactoMineR. R package version 1.26. [p151]

R. A. Johnson and D. W. Wichern. Applied Multivariate Statistical Analysis. Prentice Hall, New Jersey, third edition, 1992. [p152, 153]

R. E. Kass, U. T. Eden, and E. N. Brown. Analysis of Neural Data. Springer, 2014. [p156]

S. Korkmaz, D. Goksuluk, and G. Zararsiz. MVN: Multivariate Normality Tests, 2014. URL http: //www. biosoft. hacettepe. edu. tr/MVN/. R package version 3.7. [p151]

K. V. Mardia. Measures of multivariate skewness and kurtosis with applications. Biometrika, 57(3): 519-530, 1970. [p152]

K. V. Mardia. Applications of some measures of multivariate skewness and kurtosis in testing normality and robustness studies. Sankhyā: The Indian Journal of Statistics, Series B (1960-2002), 36(2):115-128, 1974. [p152]

C. J. Mecklin and D. J. Mundfrom. On using asymptotic critical values in testing for multivariate normality. InterStat, 1:1-12, 2003. [p152]

C. J. Mecklin and D. J. Mundfrom. A monte carlo comparison of the type I and type II error rates of tests of multivariate normality. Journal of Statistical Computation and Simulation, 75(2):93-107, 2005. [p151, 153] 
S. Ramzan, F. M. Zahid, and S. Ramzan. Evaluating multivariate normality: A graphical approach. Middle East Journal of Scientific Research, 13(2):254-263, 2013. [p151]

W. Revelle. psych: Procedures for Psychological, Psychometric, and Personality Research. Northwestern University, Evanston, Illinois, 2014. URL http://CRAN. R-project. org/package=psych. R package version 1.4.8. [p151]

G. J. S. Ross. MLP, Maximum Likelihood Program. Harpenden: Rothamsted Experimental Station, 1980. [p153]

P. J. Rousseeuw and A. M. Leroy. Robust Regression and Outlier Detection. John Wiley \& Sons, Inc., New York, NY, USA, 1987. ISBN 0-471-85233-3. [p158]

J. P. Royston. An extension of Shapiro and Wilk's W test for normality to large samples. Applied Statistics, 31(2):115-124, 1982. [p153]

J. P. Royston. Some techniques for assessing multivariate normality based on the Shapiro-Wilk W. Applied Statistics, 32(2):121-133, 1983. [p153]

P. Royston. Approximating the Shapiro-Wilk W test for non-normality. Statistics and Computing, 2(3): 117-119, 1992. [p153]

P. Royston. Remark as r94: A remark on algorithm AS 181: The W test for normality. Applied Statistics, 44(4):547-551, 1995. [p153]

RStudio, Inc. shiny: Web Application Framework for R, 2014. URL http://CRAN.R-project.org/ package=shiny. R package version 0.10.1. [p159]

S. S. Shapiro and M. B. Wilk. An analysis of variance test for normality (complete samples). Biometrika, 52:591-611, 1964. [p153]

J. P. Stevens. Applied multivariate statistics for the social sciences. Routledge, 2012. [p156]

T. Svantesson and J. W. Wallace. Tests for assessing multivariate normality and the covariance structure of mimo data. In Acoustics, Speech, and Signal Processing, 2003. Proceedings.(ICASSP'03). 2003 IEEE International Conference on, volume 4, pages 656-659. IEEE, 2003. [p151]

W. N. Venables and B. D. Ripley. Modern Applied Statistics with S. Springer, New York, fourth edition, 2002. URL http://www. stats.ox.ac.uk/pub/MASS4. ISBN 0-387-95457-0. [p151]

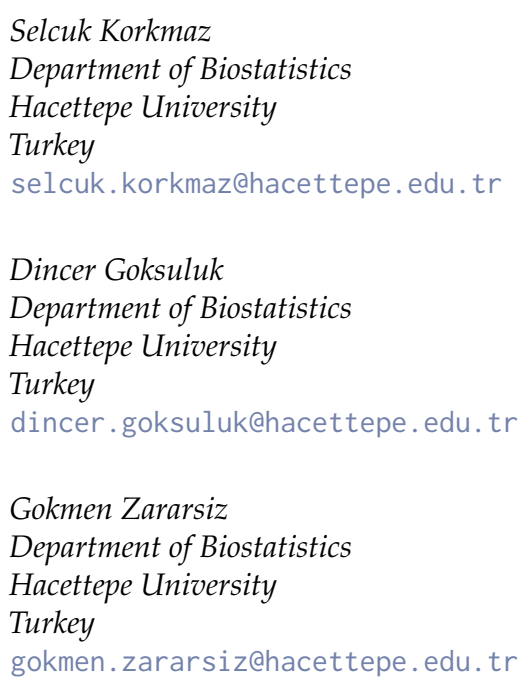

